\title{
Multidrug therapy for leprosy in Trinidad and Tobago: a preliminary report
}

\author{
R F KEELER \\ Hansen's Disease Control Unit, Ministry of Health and Environ- \\ ment, 182 Western Main Road, Cocorite via St James, Trinidad, \\ West Indies
}

Accepted for publication 18 April 1984

\begin{abstract}
Summary Dual chemotherapy for multibacillary patients was introduced in Trinidad and Tobago in 1971, using clofazimine and dapsone. Since 1973, newly-diagnosed multibacillary patients have received triple therapy, at first only for a few weeks, but later for 3 months, adding rifampicin $600 \mathrm{mg}$ daily to the clofazimine and dapsone already in use. In January 1982, the short-course regimens recommended by the World Health Organization (WHO) (Technical Report series, 1982) were introduced and after a period of 21 months, 531 patients had completed their courses of treatment. This paper reports preliminary results in this group.
\end{abstract}

\section{Introduction}

Trinidad and Tobago is the southern-most island nation of the Caribbean Sea, lying close to the South American coast of Venezuela. This nation covers an area of $5128 \mathrm{~km}^{2}$ and has a population of 1.1 million. Its inhabitants are primarily of East Indian and African descent in nearly equal numbers.

In 1969 the Ministry of Health abolished a policy of admitting newly-diagnosed leprosy patients to its leprosarium on Chacachacare Island, and in 1978 the Government took a decision to close the leprosarium and to find suitable accommodation in the community for the inmates. Patients have since then been treated successfully in existing health centres throughout the nation.

Dual chemotherapy for multibacillary patients was introduced in 1971 using clofazimine $100 \mathrm{mg}$ and dapsone $100 \mathrm{mg}$ daily in adults. Since 1973, newly-diagnosed multibacillary patients have been given triple drug therapy, at first for only a few weeks, but later for 3 months, adding rifampicin $600 \mathrm{mg}$ daily to the clofazimine and dapsone already in use. After the initial 3 months of therapy, the latter two drugs were continued until the patient was bacteriologically negative 
over a period of at least 12 months, after which dapsone monotherapy was maintained indefinitely.

A vertical programme, the Hansen's Disease Control Unit, was established in 1971 with emphasis on case finding and case holding in an out-patient setting.

Three years after the unit was established, the new case rate peaked at twice the level in year 1, and it has steadily fallen since then to its present low level which is half the new case rate of year 1, or one-quarter of the peak new case rate of year 3 .

Corresponding with the fall in the new case rate there has been a fall in the number of bacteriologically-positive patients (from a peak of 188 in 1974 to 35 at the end of 1982) indicating that: 1, chemotherapy was successful; and 2, the reservoir of Mycobacterium leprae was being reduced, thus enabling a chain of transmission to be broken.

Unfortunately we have had unacceptably high rates of non-compliance over the years, especially after the skin lesions disappeared. Too many patients defaulted before completing the recommended period of time needed to take a patient's name off the register and to consider him as released from control. Although we have had very few (3) cases of secondary drug resistance to dapsone and no known cases of primary drug resistance, we were therefore greatly interested to learn of the World Health Organization (WHO) short course, multidrug, partially supervised regimens, ${ }^{1}$ primarily because of our compliance problems and heavy workload. Having adequate personnel, medication and mobility, we decided to start the WHO regimens in January 1982, making them available to all patients on our register, and to new patients on diagnosis.

\section{Patients and methods}

As of 1 January 1982 we had 718 patients on our register. Thirty new patients were added in 1982 and 17 new patients were added during the first 9 months in 1983. After 21 months of following this regimen, 531 patients have completed their course of therapy.

Our standard drugs were dapsone, clofazimine, rifampicin and DADDS. For 3 patients who had proven or clinically suspected secondary drug resistance to dapsone we used ethionamide instead of dapsone and DADDS. For 1 who refused to take clofazimine we used ethionamide as the alternative drug. Our standard regimen for all patients, whether having paucibacillary or multibacillary disease, was as follows (for $70 \mathrm{~kg}$ adults): once a month, under supervision: rifampicin $600 \mathrm{mg}$, clofazimine $300 \mathrm{mg}$, dapsone $100 \mathrm{mg}$, DADDS $450 \mathrm{mg}$, i.m.; daily, self-administered: clofazimine $100 \mathrm{mg}$, dapsone $100 \mathrm{mg}$. We decided to add DADDS and to give dapsone under observation monthly, in addition to the rifampicin and clofazimine, because of our concern regarding compliance. Fortunately, there is minimal objection in Trinidad and Tobago to the temporary 
cutaneous discolouration caused by clofazimine. However, there is moderate objection to the use of DADDS, primarily because of the pain associated with its deposition in the gluteal muscles.

We gave 6 months of multidrug therapy to patients with paucibacillary disease who were still on our register, whether their disease was active or inactive. We also gave only 6 months of multidrug therapy to patients with multibacillary disease if they had been bacteriologically negative (no AFB seen on slit smear) for 4 years or more prior to the commencement of this new regimen. We gave 12 months of multidrug therapy to patients with multibacillary disease if they had been bacteriologically negative for at least 2 years, but for less than 4 years. If patients with multibacillary disease were bacteriologically positive at any time within the preceding 2 years, they were scheduled for 24 months of multidrug therapy.

Patients were examined and given treatment at regularly held leprosy clinics in 10 health centres scattered throughout the island until 31 March 1983, when 3 of the smaller clinics were discontinued due to a lack of patients needing medication. In addition, institutionalized, aged, isolated and homebound patients were seen at their places of residence for similar care by our District Health Visitors with periodic visits also being made by our medical officers. Our field staff is composed of 2 physicians, 1 medical social worker, 1 laboratory technician, 1 physiotherapist, 1 orthopaedic shoemaker, 4 nurses (District Health Visitors), and 5 follow-up workers.

Patients who were non-compliant were pursued by letters and by personal visits. Attempts were made to meet their unique needs, even if this meant providing a time and place for examination and treatment outside of the traditional working hours and venues. As a result many received chemotherapy who otherwise would have remained out of control.

We plan to re-examine the patients who have had paucibacillary disease at 6-month intervals for 3 years prior to ending their surveillance. Patients with multibacillary disease will be re-examined also at 6-month intervals, but for a total of 5 years before terminating their surveillance.

\section{Results}

There was excellent acceptance of the multidrug regimen by our patients. Only 3 refused to take the monthly supervised dose of medication. Nineteen others continued to ref use to take any anti-leprosy medication. We have been unable to contact 18 of our registered patients. Five patients complained of adverse responses to the multidrug, supervised regimen and 4 of these refused further treatment. The patient who continued with treatment was a 58 -year-old East Indian female who reported a rash which appeared about an hour after taking her supervised medication. This occurred twice, following her second and third 
monthly visits, and in each instance disappeared in 24 hours. Only clofazimine and dapsone were given on her fourth monthly visit. No cutaneous reaction was noted. Rifampicin was reintroduced on the fifth monthly visit with no adverse response. She continued then on the normal multidrug regimen until her course was completed without additional adverse responses.

During the first 21 months of pursuing the WHO's recommended multidrug, partially supervised, short-course regimen 531 patients completed their chemotherapy.

Of the 718 on the register on 1 January 1982, 514 completed their chemotherapy. Thus $72 \%$ of patients on the register when this regimen commenced have completed their treatment. Fifteen others were patients diagnosed in 1982, and an additional 2 were diagnosed in 1983.

A breakdown of the 531 patients reveals a slight preponderance of males $(54 \%)$, a low proportion of children $(6 \%)$, a high proportion of patients with a diagnosis made more than 10 years ago $(60 \%)$, and a nearly equal division into paucibacillary and multibacillary groups (see Tables 1-4).

We had a higher percentage of males than of females in our original group of 718 , so we were not surprised to find a slight preponderance of males a mong those who had completed their chemotherapy.

Regarding children, less than $8 \%$ of our registered patients at the beginning of this multidrug programme were under the age of 15 years, so a low proportion of children was expected out of the 531 patients who completed their chemotherapy. All 31 children who completed their chemotherapy were in the 5-14-year age group.

Of the $40 \%$ whose diagnoses were made within the last 10 years, 74 patients $(14 \%$ of the 531$)$ had their diagnoses made during the last 5 years.

Since $51 \%$ of those completing the multidrug regimen had multibacillary

Table 1. Patients completing multidrug regimen

\begin{tabular}{lc}
\hline \multicolumn{1}{c}{ Sex } & Number (\%) \\
\hline Male & $288(54)$ \\
Female & $243(46)$ \\
\hline Total & 531 \\
\hline
\end{tabular}

Table 2. Patients completing multidrug regimen (age)

\begin{tabular}{cc}
\hline $\begin{array}{c}\text { Age group } \\
\text { (years) }\end{array}$ & Number $(\%)$ \\
\hline $0-4$ & 0 \\
$5-14$ & $31(6)$ \\
$15-24$ & $91(17)$ \\
$25-44$ & $131(25)$ \\
$45-64$ & $172(32)$ \\
$65-84$ & $104(20)$ \\
$85+$ & $2(<1)$ \\
\hline Total & 531 \\
\hline
\end{tabular}


Table 3. Patients completing multidrug regimen (time since diagnosis)

\begin{tabular}{cc}
$\begin{array}{c}\text { Years since } \\
\text { diagnosis }\end{array}$ & Number $(\%)$ \\
\hline $0-9$ & $213(40)$ \\
$10-19$ & $149(28)$ \\
$20-29$ & $78(15)$ \\
$30-39$ & $59(11)$ \\
$40-49$ & $26(5)$ \\
$50-59$ & $5(1)$ \\
$60-69$ & $1(<1)$ \\
$70+$ & 0 \\
\hline Total & 531 \\
\hline
\end{tabular}

Table 4. Patients completing multidrug regimen (classification)

\begin{tabular}{lrr}
\hline \multicolumn{1}{c}{ Classification } & Number & \\
\hline Paucibacillary & & \\
$\quad$ Indeterminate & 9 & \\
Tuberculoid (TT) & 124 & \\
Borderline (BT) & 127 & \\
\cline { 2 - 3 } Sub-total: & 260 & $(49 \%)$ \\
Multibacillary & & \\
$\quad$ Borderline (BB) & 50 & \\
Borderline (BL) & 47 & \\
Lepromatous (LL) & 174 & \\
Sub-total: & 271 & $(51 \%)$ \\
\hline Total & 531 & \\
\hline
\end{tabular}

disease and since in the past we tried to keep multibacillary patients on chemotherapy for a lifetime it is not surprising to see that over $30 \%$ of the patients had been diagnosed 20 years ago or more, and that $52 \%$ are over age 44 .

One 44-year-old obese female with tuberculoid disease has retained her hypopigmented lesion up to the present time (15 months after her 6 months of multidrug therapy was completed). A repeat biopsy revealed resolving granulomas, but no evidence of active disease.

Other patients with tuberculoid disease who had remaining faint hypopigmentation at the time chemotherapy was discontinued at 6 months, have all subsequently re-pigmented well, with no recurrence so far. Nine paucibacillary patients were given several months additional treatment because of prominent lesions of unresolved neuritis at the 6 months point.

Since the completion of therapy 1 patient has migrated to the United States of America, and 3 patients have died, leaving 527 for surveillance. None of the 3 deaths was related to their chemotherapy.

There was an initial increase in the attendance at clinics in early 1982, but since the latter half of 1982 there has been a considerable decline. As a result 3 smaller clinics have been closed. Patients from these areas can attend 1 of the remaining 7 clinics for their semi-annual evaluations over the next 3-5 years. Another clinic was closed 1 April 1984. Hopefully, leprosy and dermatological services could be combined in the remaining strategically located centres for the future.

Lesions definitely respond much more rapidly, clinically, in patients on multidrug therapy in which rifampicin is included than in monotherapy or dual therapy where rifampicin is absent. Easily detectable improvement occurs in less 
than 2 weeks in most patients. This is encouraging to the patient as well as to the physician.

There has not been any increase in the frequency or in the severity of reactions seen in patients taking this multidrug regimen. No disabilities have developed during or after the period of chemotherapy.

\section{Discussion}

It is too early to accurately evaluate the effectiveness of this multidrug regimen in Trinidad and Tobago. In another $2-3$ years, however, I feel we should be able to satisfactorily evaluate its effectiveness in patients with paucibacillary disease. It will probably take a bit longer to adequately evaluate its effectiveness in patients with multibacillary disease.

Most patients are happy to be able to cease taking medication after a specified, relatively short period of time; a few, however, want to remain on medication indefinitely.

With three-quarters of our patients having completed their chemotherapy our staff can now concentrate more effectively on newly-diagnosed patients, bacteriologically-positive patients, non-complying patients and significant contacts. We are now able to tackle our high priorities more effectively and help our patients sense that having leprosy need only be a chapter in their lives. Their outlook on life has improved considerably as they anticipate freedom from medication and from disease in the future.

\section{References}

1 WHO. Chemotherapy of Leprosy for Control Programmes. Report of a WHO Study Group. (Technical Report series 675). Geneva: WHO, 1982. 